\title{
Comparison of two- and three-dimensional measurement of the Cobb angle in scoliosis
}

\author{
Ricarda Lechner ${ }^{1}$ David Putzer ${ }^{2}$ - Dietmar Dammerer ${ }^{1}$ - Michael Liebensteiner ${ }^{1}$. \\ Christian Bach ${ }^{3} \cdot$ Martin Thaler ${ }^{1}$
}

Received: 5 September 2016 / Accepted: 21 November 2016 / Published online: 5 December 2016

(C) The Author(s) 2017. This article is published with open access at Springerlink.com

\begin{abstract}
Purpose The Cobb angle as an objective measure is used to determine the progression of deformity, and is the basis in the planning of conservative and surgical treatment. However, studies have shown that the Cobb angle has two limitations: an inter- and intraobserver variability of the measurement is approximately 3-5 degrees, and high variability regarding the definition of the end vertebra. Scoliosis is a three-dimensional (3D) pathology, and 3D pathologies cannot be completely assessed by two-dimensional (2D) methods, like 2D radiography. The objective of this study was to determine the intraobserver and interobserver reliability of end vertebra definition and Cobb angle measurement using X-rays and 3D computer tomography (CT) reconstructions in scoliotic spines.

Methods To assess interoberver variation the Cobb angle and the end vertebra were assessed by five observers in 55 patients using X-rays and 3D CT reconstructions. Definition of end vertebra and measurement of the Cobb angle was repeated two times with a three-week interval. Intraclass correlation coefficients (ICC) were used to determine the interobserver and intraobserver reliabilities. 95\% prediction limits were provided for measurement errors.

Results Intraclass correlation coefficient (ICC) showed excellent reliability for both methods. The measured Cobb angle
\end{abstract}

Martin Thaler

martin.thaler@i-med.ac.at

1 Department of Orthopaedic Surgery, Medical University Innsbruck, Anichstr. 35, 6020 Innsbruck, Austria

2 Department of Orthopaedic Surgery - Experimental Orthopaedics, Medical University Innsbruck, Innrain 36, 6020 Innsbruck, Austria

3 Department of Orthopaedic Surgery, Landeskrankenhaus Feldkirch, Carinagasse 47, 6807 Feldkirch, Austria was on average 9.2 degrees larger in the 3D CT group ( $72.8^{\circ}$, range $30-144)$ than on $2 \mathrm{D}$ radiography $\left(63.6^{\circ}\right.$, range $\left.24-152\right)$. Conclusions In scoliosis treatment it is very essential to determine the curve magnitude, which is larger in a 3D measurement compared to $2 \mathrm{D}$ radiography.

Keywords Cobb angle $\cdot$ Scoliosis $\cdot$ Three-dimensional measurement $\cdot$ Interobserver, intraobserver reliability

\section{Introduction}

Accuracy and consistency are crucial in the radiographic assessment of scoliosis. The findings of these measurements have significant implications for the treatment and management of patients [1]. Decisions to observe, brace, or recommend surgical intervention for scoliosis are based in large part on radiographic criteria, especially the Cobb angle [2]. The Cobb method has become the standard parameter for quantifying scoliosis curve magnitude [3]. However, the Cobb method has some limitations. Studies of interobserver and intraobserver reliability in measurement of the Cobb angle have shown a measurement error of approximately $3-5^{\circ}$ [3-6]. Sources of error may include a wrong definition of end vertebra, an incorrect drawing of the lines through end plates or through the pedicles, and drawing of perpendiculars or the measurement of the angle itself [7]. The Cobb angle is usually assessed from anterior-posterior or posterior-anterior standing radiographs. However, scoliosis is a threedimensional (3D) deformity, whereas the Cobb angle measured in a plane X-ray is a two-dimensional (2D) value. 3D pathologies cannot be completely assessed by $2 \mathrm{D}$ methods, like radiography. The direction of the X-ray beam cannot be adjusted to display the largest extent of the curve. Thus, accurate measurement of the radiological extent of the deformity is 
hardly possible. New software technologies and the improvement in CT accuracy have made it possible to generate anatomically accurate and detailed 3D reconstructions of the vertebral column. The aim of our study was to investigate Cobb angle measurement in 3D reconstructed images of the spine and to compare them with conventional supine radiographs, as well as to determine the interobserver and intraobserver variability associated with both techniques.

\section{Materials and methods}

Anterior-posterior digital full-length spine supine radiographs and $\mathrm{CT}$ scans of the vertebral column were performed in 55 patients with idiopathic and neuropathic scoliosis. Therefore the patients' feet were positioned in a sub-talar joint neutral position with each foot on the ground and standing in a relaxed manner. The chin was directed as if to avoid its shadow on the spinal X-ray radiographs. Furthermore, the arms while standing were maintained straight in relaxed position on both sides.

A General Electrics Lightspeed $16^{\odot}$ (GE Healthcare, Wisconsin, USA) was used with $100 \mathrm{kV}, 100 \mathrm{~mA}$ source, rotation $0.8 \mathrm{sec}$, DFOV 15, noise index 20 and slice thickness of $0.625 \mathrm{~mm}$. Scan coverage was vertebral levels $\mathrm{C} 1$ to S1.

Reformatted three-dimensional (3D) reconstructions were produced from the axial CT slides using the Advantage imaging processing software (Advantage Windows V4.01, GE Medical Systems). The 3D reconstructions of the whole spine can then be rotated virtually in any direction (Fig. 1). Therefore, the largest extent of every single scoliotic curve can be assessed. Five observers, including two experienced spine surgeons, two spine fellows and one senior paediatric orthopaedic surgeon were instructed to measure the Cobb angle. They were all familiar with the measurement method of the Cobb angle and carried out the measurements independently twice in each setting (measurement on digital radiographs, measurement of the $3 \mathrm{D}$ reconstructions), with a three-week interval between each session. All observers were blinded to their prior measurements and to the other observers. In a first step end vertebrae were defined in the radiographs and the $3 \mathrm{D}$ reconstructions. In a second step the Cobb angles were measured with predefined end vertebrae. Therefore, the upper and lower end vertebrae of each curve were defined by the senior author by measuring the maximum inclination angle on the same radiographs and 3D images. Lines were drawn through the end plates of each vertebra, and the one with the largest angle to the horizontal was defined as an end vertebra. The radiographs as well as the $3 \mathrm{D}$ reconstructions were all blinded and numbered. The measurements were carried out using the Advantage Windows software. For the 3D reconstructions of the vertebral column the observers were asked to rotate the curve to the largest extent of curve magnitude. The

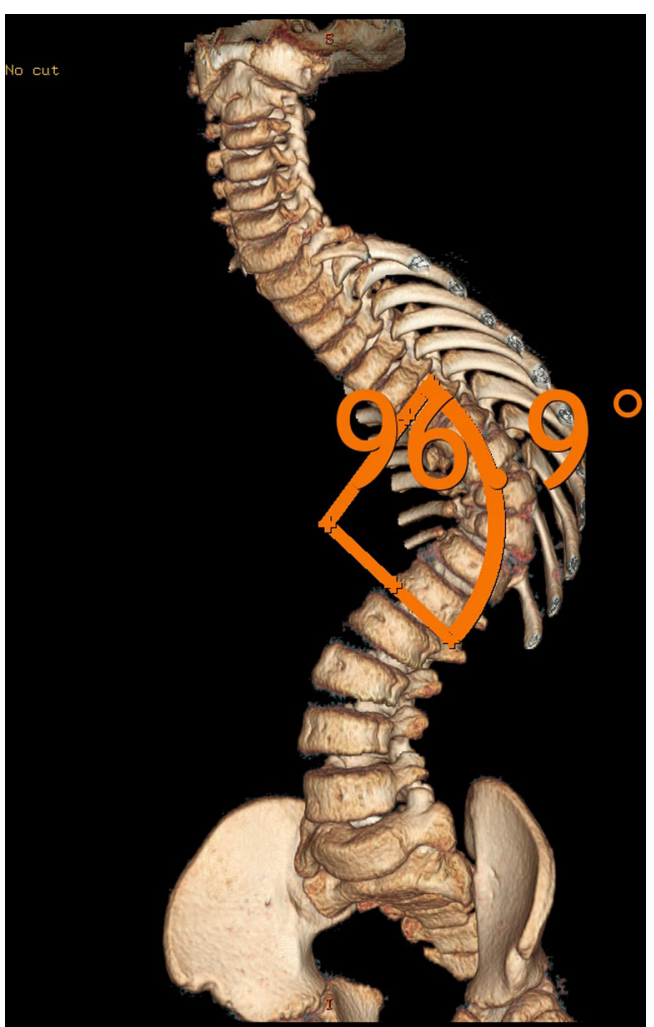

Fig. $13 \mathrm{D}$ reconstruction of the whole spine rotated to the largest extension of the scoliotic curve. The Cobb angle was measured with the end plates perpendicular to the plane of the viewer. The Cobb angle is approximately $20^{\circ}$ larger compared to conventional radiography

end plates of the end vertebra had to be a single clear line perpendicular to the view of the observer. Then the Cobb angles were measured. For Cobb angle measurement lines were drawn through the end plates of the upper and lower end vertebrae of the curve. The program measured the Cobb angle automatically.

\section{Statistical analysis}

The intraclass correlation coefficient (ICC) two-way mixed model on absolute agreement was used to analyze measurement reliability [8]. The values of the ICC can range from 0 to 1 , with a higher value indicating better reliability. ICC less than 0.40 was considered as poor, $0.40-0.59$ as fair, 0.60 0.74 as good, and $0.75-1.00$ as excellent [8]. The deviations of mean values, as well as maximum and minimum values were calculated with descriptive statistics. Additionally, summary statistics from analyses-of-variance calculations were used to provide $95 \%$ prediction limits for the error in measurements. The Cobb angle, upper vertebra, and lower vertebra for conventional radiography and $3 \mathrm{D}$ reconstructions groups were compared for significant differences using the Wilcoxon test for non-parametric data. Statistical analyses were performed using SPSS 12.0 software (SPSS Inc., Chicago, IL, USA). 
Table 1 Curve characteristics of conventional and digital radiography groups

\begin{tabular}{llll}
\hline Characteristic & 2D plain radiographs & 3D CT reconstructions & P-value \\
\hline Number $(n)$ & 55 & 55 & \\
Cobb angle (range) & $63.64(24-152)$ & $72.76(30-144)$ & $<0.001^{*}$ \\
Number $(n)$ & 41 & 41 & 0.221 \\
Upper vertebra (range) & T3-T12 & T1-T12 & $0.004 *$ \\
Lower vertebra (range) & T9-L5 & T10-L5 & \\
\hline
\end{tabular}

Data for measurements by Observer 1 using conventional radiography and $3 \mathrm{D}$ reconstructions. $P$-value calculated using the Wilcoxon test (* statistically significant)

\section{Results}

Significant differences between the two methods were found for the Cobb angle and lower vertebra (Table 1). The mean Cobb angle was 63.6 (range 24-152) for all radiographs and 72.8 (range 30-144) for all 3D reconstructions. This difference was statistically significant $(p<0.001)$. The distribution of curve angles is shown in Table 2. For the definition of end vertebrae and Cobb angle, the intraobserver ICC was excellent in the radiographs and in the 3D reconstructions, except for the definition of the lower end vertebra of observer 2 in the $3 \mathrm{D}$ reconstructions (Table 3 ). Lowest variability ( 0.53 levels) for the upper proximal vertebra was found for radiographs (Table 4). Interobserver ICC was excellent for all parameters except for lower vertebra in the 3D reconstructions $(0.74)$. Interobserver reliability for the Cobb angle was excellent (radiographs, $0.93-0.97 ; 3 \mathrm{D}$ reconstructions, $0.92-0.96$ ) for both methods. Variability of Cobb angles was similar for both methods, but the minimum and maximum values had a larger range in the $3 \mathrm{D}$ reconstructions $\left(3.57^{\circ}\right.$; range $1.36-10.64 /$ radiographs, $3.62^{\circ}$; range $0.72-6.96$ ).

\section{Discussion}

Recent decades have witnessed significant advances in the treatment of scoliosis. Critical to these advancements has been the progress made in determination of radiological parameters [9]. Scoliosis is a complex 3D anomaly of the spine including deviations in the frontal plane, modifications of the sagittal profile, rotations in the transverse plane, and alterations of the rib cage [10]. Owing to the introduction of digital radiography, important parts of the spine can be enlarged and seen more clearly by modifying the contrast, and the margins of the vertebrae can be enhanced by computerized options. Despite this technical support, an inaccuracy in angle measurement persists due to a disadvantage of the Cobb method itself. Inter- and intraobserver variabilities of approximately $3-5^{\circ}$ have been published $[4,7]$.

Another systematic bias is that there is a high variability in end vertebra selection. We still measure a two-dimensional value of a three-dimensional structure. The end plate does not have a trajectory as a single clear line when the angle of its plane is other than perpendicular to the plane of the film. Even in the best conditions, an end plate that appears fusiform on the screen or on the film cannot be reduced to a single line [7]. Since each vertebra has a distinct rotational component, it is impossible to select a plane in X-rays in which the scoliotic region has a true anterior-posterior or posterior-anterior view of profile projection [11]. Especially, in combined deformities, like kyphoscoliosis or lordoscoliosis, the determination of the true Cobb angle is nearly impossible with conventional radiography [10] (Figs. 1 and 2). Stagnara stated that in cases of severe scoliosis the apical vertebral rotation was so appreciable that the upper vertebrae were seen only on the lateral view in the anteroposterior radiograph [12]. In some cases the kyphosis detected on the lateral radiograph is not real kyphosis, but the frontal curve of the scoliotic spine that has been
Table 2 Distribution of curve angles (Cobb method) in the 55 patients of the study cohort

\begin{tabular}{|c|c|c|c|c|c|}
\hline \multirow[t]{2}{*}{ Cobb angle $\left(^{\circ}\right)$} & \multicolumn{2}{|c|}{ 2D plain radiographs } & \multicolumn{2}{|c|}{ 3D CT reconstructions } & \multirow[t]{2}{*}{ Difference } \\
\hline & Curves & Percent $(\%)$ & Curves & Percent $(\%)$ & \\
\hline $20-40$ & 7 & 12.7 & 4 & 7.3 & -3 \\
\hline $41-60$ & 18 & 32.7 & 13 & 23.6 & -5 \\
\hline $61-80$ & 22 & 40.0 & 20 & 36.4 & -2 \\
\hline $81-100$ & 4 & 7.3 & 13 & 23.6 & +9 \\
\hline $101-120$ & 1 & 1.8 & 2 & 3.6 & +1 \\
\hline$\geq 121$ & 3 & 5.5 & 3 & 5.5 & +0 \\
\hline Total & 55 & 100 & 55 & 100 & \\
\hline
\end{tabular}




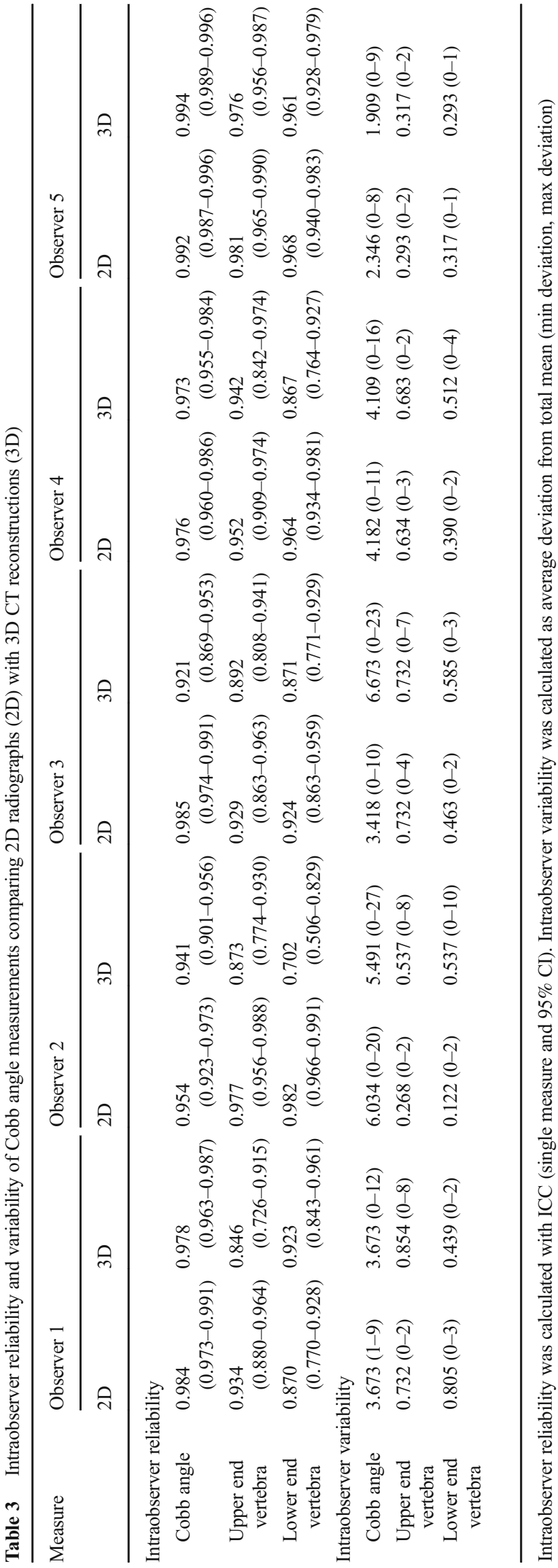

Table 4 Interobserver reliability and variability of Cobb angle measurements by radiographic method

Measure

2D plain radiographs $3 \mathrm{D} \mathrm{CT}$ reconstructions

Interobserver reliability

Cobb angle

$0.956(0.928-0.973) \quad 0.943(0.917-0.963)$

Upper vertebra

$0.850(0.778-0.907) \quad 0.857(0.786-0.912)$

Lower vertebra

$0.832(0.753-0.895) \quad 0.744(0.637-0.836)$

Interobserver variability

Cobb angle in degrees $3.620(0.72-6.96)$

$3.571(1.36-10.64)$

Upper vertebra

$0.523(0.00-2.64)$

$0.581(0.00-2.88)$

Lower vertebra

$0.659(0.00-3.52)$

$0.587(0.00-3.04)$

Interobserver reliability was calculated with ICC (single measure and $95 \% \mathrm{CI}$ ). Interobserver variability was calculated as average deviation from total mean (min deviation, max deviation)

displaced into the sagittal plane as a result of the appreciable vertebral rotation [12].

Thus, the radiographic image is just a simple, mere projection or a shadow of a three-dimensional structure. The widespread use of CT represents probably the single most important advance in diagnostic radiology. The continuing improvement in CT techniques and software has made 3D CTreconstructed images a new tool for assessing 3D deformities. 3D reconstructed CT images are well-established diagnostic methods in vascular, transplantation and neuro-surgery [13-15].

Although CT radiation doses still limit their use for routine repeated scoliosis assessment, in very few cases a single preoperative $\mathrm{CT}$ scan for surgical planning may be justifiable. In some hospitals a single preoperative CT scan is performed in cases of patients undergoing complex scoliosis surgery

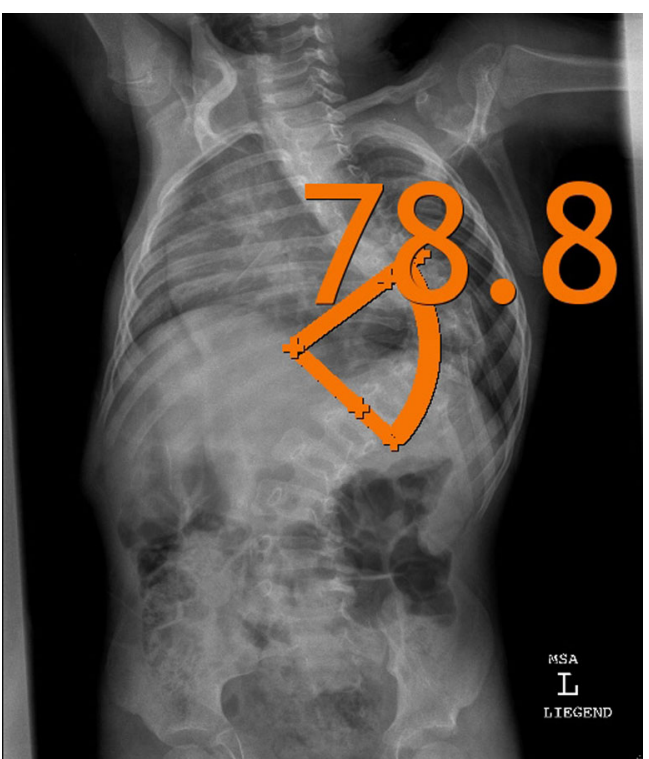

Fig. 2 Showing the conventional measurement of a Cobb angle by means of an X-ray. The Cobb angle measured here is approximately $20^{\circ}$ smaller compared to the $3 \mathrm{D}$ reconstructions of the whole spine 
$\left(\mathrm{Cobb}>100^{\circ}\right)[16]$. In these cases appropriate decisionmaking is essential as to whether the pedicle diameters are large enough for screw placement. A preoperative CT scan allows for safer screw sizing and positioning because it helps to assess the pedicle diameter [17]. The remaining CT reconstructions measured in the present study were carried out for other medical reasons than surgical planning. Recently published data have shown that the estimated radiation dose for a 3D CT reconstruction can be reduced to 3.7 millisieverts (mSv) [18].

3D measurements can also be evaluated with the help of the EOS software imaging system [19]. This system is based on posteroanterior and lateral views of the spine. These authors also showed that routine 2D measurements of deformities underestimate the amount of Cobb angle because of the axial plane rotation. These techniques have the advantage that a software calculates a $3 \mathrm{D}$ reconstruction of the spine based on radiographs of the standing patient $[20,21]$.

We aimed to compare the intraobserver and interobserver reliability in the definition of end vertebrae, as well as in Cobb angle measurement in radiographs of scoliotic spines comparing digital radiographs and in 3D CT images. We found no significant difference in the intraobserver or interobserver reliability between conventional radiography and 3D CT images in Cobb angle measurement and determination of the end vertebrae. The intraobserver reliability for the Cobb angle found in this study was nearly the same for both methods (mean ICC radiographs, 0.978; mean ICC 3D CT images, 0.961 ). The variability and reliability of Cobb angle measurement in 3D CT images described in the present study are comparable to the results previous studies reported for manual or digital measurement of plain radiographs [7, 22]. We measured the Cobb angle with predefined end vertebrae because it has been shown that the reliability is higher when the error of end plate selection is limited [7].

A limitation of the present study is that Cobb angles measured from a supine CT cannot be directly compared with fulllength standing radiographs due to changes in spinal geometry. Torell et al. reported an average deviation of $8.8^{\circ}$ comparing standing and supine radiographs [23]. A decrease of the average Cobb angle from $56^{\circ}$ to $39^{\circ}$ regarding standing and supine position was shown by Yazici et al. [24]. Therefore, we compared the 3D CT images with supine full-length spine radiographs. Carman, using conventional radiographs, found that a difference of approximately $10^{\circ}$ had to be present to be 95\% confident that a true change in spinal geometry had occurred [3]. We found a mean difference of $9.2 \%$ between 3D $\mathrm{CT}$ images and radiographs $(p<0.005)$ (Table 2$)$. The explanation for the difference during $3 \mathrm{D}$ measurements might be that the observer could rotate the $3 \mathrm{D}$ reconstruction of the spine $360^{\circ}$ to measure the Cobb angle (Figs. 1 and 2). In daily clinical routine, conclusions about the severity of scoliosis and risk of progression are often drawn from the Cobb angle measurement of the major curve on the standing coronal image.

Another limitation of our study is that CT radiation doses currently preclude CT use in routine clinical practice and Cobb angle measurement in non-complex scoliosis. However, future advances in multislice CT will allow for lower dosages and faster acquisition times, thus enabling the increased use of CT for the assessment of scoliotic deformities. Currently the dosage can be reduced to $3.7 \mathrm{mSv}$ [16].

Our data suggest that the Cobb angle of the scoliosis might be significantly larger than usually measured by plain X-rays. This should be especially considered in decision-making for conservative or surgical treatment in borderline scoliosis. We believe that, in comparison with conventional radiography, our 3D CT images of the spine feature a higher resolution and yield superior results regarding the "real" Cobb angle. Low inter- and intraobserver reliability and high variabilities in end vertebra definition are the reported major limitations of the Cobb angle. The difference of approximately $10^{\circ}$ between $3 \mathrm{D}$ and $2 \mathrm{D}$ measurement found in the present study demonstrates another limitation of the Cobb angle.

Acknowledgments Open access funding provided by University of Innsbruck and Medical University of Innsbruck.

\section{Compliance with ethical standards}

Conflict of interest The authors declare that they have no conflict of interest.

Funding This study was funded with internal funds from the research institution. No payments were received for this study.

Ethical approval Ethical approval was not required for this study.

Open Access This article is distributed under the terms of the Creative Commons Attribution 4.0 International License (http:// creativecommons.org/licenses/by/4.0/), which permits unrestricted use, distribution, and reproduction in any medium, provided you give appropriate credit to the original author(s) and the source, provide a link to the Creative Commons license, and indicate if changes were made.

\section{References}

1. Colwell HR (1990) Radiographic measurements and clinical decisions. J Bone Joint Surg Am 72(3):319

2. Cobb JR (1948) Outlines for the study of scoliosis. Instr Course Lect 5:261-275

3. Carman DL, Browne RH, Birch JG (1990) Measurement of scoliosis and kyphosis radiographs. Intraobserver and interobserver variation. J Bone Joint Surg Am 72(3):328-333

4. Geijer H, Beckman K, Jonsson B, Andersson T, Persliden J (2001) Digital radiography of scoliosis with a scanning method: initial evaluation. Radiology 218(2):402-410

5. Geijer H, Verdonck B, Beckman KW, Andersson T, Persliden J (2003) Digital radiography of scoliosis with a scanning method: 
radiation dose optimization. Eur Radiol 13(3):543-551. doi:10.1007/s00330-002-1476-1

6. Gross C, Gross M, Kuschner S (1983) Error analysis of scoliosis curvature measurement. Bull Hosp Jt Dis Orthop Inst 43(2):171-177

7. Gstoettner M, Sekyra K, Walochnik N, Winter P, Wachter R, Bach CM (2007) Inter- and intraobserver reliability assessment of the Cobb angle: manual versus digital measurement tools. Eur Spine J 16(10):1587-1592. doi:10.1007/s00586-007-0401-3

8. Shrout PE, Fleiss JL (1979) Intraclass correlations: uses in assessing rater reliability. Psychol Bull 86(2):420-428

9. Potter BK, Rosner MK, Lehman RA Jr, Polly DW Jr, Schroeder TM, Kuklo TR (2005) Reliability of end, neutral, and stable vertebrae identification in adolescent idiopathic scoliosis. Spine (Phila Pa 1976) 30(14):1658-1663

10. Villemure I, Aubin CE, Grimard G, Dansereau J, Labelle H (2001) Progression of vertebral and spinal three-dimensional deformities in adolescent idiopathic scoliosis: a longitudinal study. Spine (Phila Pa 1976) 26(20):2244-2250

11. DeJonge T, Dubousset JF, Illes T (2002) Hyperrotatory paradoxic kyphosis. Spine 27(4):393-398

12. Stagnara $P$ (1981) Surgical treatment of kyphotic scoliosis in the adult. Acta Orthop Belg 47(4-5):721-739

13. Min SK, Kim SY, Park YJ, Lee W, Jung IM, Lee T, Ha J, Kim SJ (2010) Role of three-dimensional computed tomography venography as a powerful navigator for varicose vein surgery. J Vasc Surg 51(4):893-899. doi:10.1016/j.jvs.2009.10.117

14. Franklin B, Gasco J, Uribe T, VonRitschl RH, Hauck E (2010) Diagnostic accuracy and inter-rater reliability of 64-multislice 3DCTA compared to intra-arterial DSA for intracranial aneurysms. J Clin Neurosci 17(5):579-583. doi:10.1016/j.jocn.2009.09.015

15. Brancatelli G, Katyal S, Federle MP, Fontes P (2002) Threedimensional multislice helical computed tomography with the volume rendering technique in the detection of vascular complications after liver transplantation. Transplantation 73(2):237-242
16. Adam CJ, Izatt MT, Harvey JR, Askin GN (2005) Variability in Cobb angle measurements using reformatted computerized tomography scans. Spine (Phila Pa 1976) 30(14):1664-1669

17. Kamimura M, Kinoshita T, Itoh H, Yuzawa Y, Takahashi J, Hirabayashi H, Nakamura I (2002) Preoperative CT examination for accurate and safe anterior spinal instrumentation surgery with endoscopic approach. J Spinal Disord Tech 15(1):47-51, discussion $51-42$

18. Schick D (2004) Computed tomography radiation doses for pediatric scoliosis scans. Internal report commissioned by Pediatric Spine Research Group from Queensland Health Biomedical Technology Services, Australia

19. Schlegl AT, Szuper K, Somoskeoy S, Than P (2015) Three dimensional radiological imaging of normal lower-limb alignment in children. Int Orthop 39(10):2073-2080. doi:10.1007/s00264-0152851-2

20. Newton PO, Fujimori T, Doan J, Reighard FG, Bastrom TP, Misaghi A (2015) Defining the "three-dimensional sagittal plane" in thoracic adolescent idiopathic scoliosis. J Bone Joint Surg Am 97(20):1694-1701. doi:10.2106/JBJS.O.00148

21. Berthonnaud E, Papin P, Deceuninck J, Hilmi R, Bernard JC, Dimnet J (2016) The use of a photogrammetric method for the three-dimensional evaluation of spinal correction in scoliosis. Int Orthop 40(6):1187-1196. doi:10.1007/s00264-015-3080-4

22. Mok JM, Berven SH, Diab M, Hackbarth M, Hu SS, Deviren V (2008) Comparison of observer variation in conventional and three digital radiographic methods used in the evaluation of patients with adolescent idiopathic scoliosis. Spine (Phila Pa 1976) 33(6):681686. doi:10.1097/BRS.0b013e318166aa8d

23. Torell G, Nachemson A, Haderspeck-Grib K, Schultz A (1985) Standing and supine Cobb measures in girls with idiopathic scoliosis. Spine (Phila Pa 1976) 10(5):425-427

24. Yazici M, Acaroglu ER, Alanay A, Deviren V, Cila A, Surat A (2001) Measurement of vertebral rotation in standing versus supine position in adolescent idiopathic scoliosis. J Pediatr Orthop 21(2):252-256 